\title{
Acropigmentación reticulada de Kitamura
}

\section{Reticulated acropigmentation of Kitamura}

\author{
María Guadalupe Olguín-García, ${ }^{\star}$ María del Carmen Palacios-Reyes, ${ }^{\ddagger}$ \\ Maribet González-González, ${ }^{\S}$ Melanie Marmolejo-Chaviraף
}

\section{RESUMEN}

En este artículo se comunica el caso de una mujer de 29 años de edad, quien acude por presentar en dorso de manos y pies múltiples manchas de color café en distintos tonos, milimétricas, bien delimitadas, que confluyen en un patrón reticular; algunas de ellas se ubican sobre áreas de atrofia. Además, se observan hoyuelos palmares (pits) que siguen e interrumpen el trayecto de los dermatoglifos. El cuadro es asintomático, benigno y corresponde a una genodermatosis de carácter autosómica dominante poco frecuente denominada acropigmentación reticulada de Kitamura (ARK).

Palabras clave: Acropigmentación reticulada de Kitamura, manchas en patrón reticular, pits palmares.

\section{ABSTRACT}

In this article, we report the case of a 29-year-old female with multiple punctate brown macules, arranged in a reticular pattern, on the dorsa of the hands and feet. Some of them are located over atrophic skin. She was also found to have pitting of the skin in both palms, following her dermatoglyphic pattern. Her clinical findings are consistent with the diagnosis of reticulate acropigmentation of Kitamura, an uncommon and benign autosomal dominant genodermatosis.

Keywords: Reticulate acropigmentation of Kitamura, palmar pits, reticular patter spots.

\section{INTRODUCCIÓN}

La acropigmentación reticulada de Kitamura (ARK), descrita por Kitamura y Akamatsu en $1943,{ }^{1}$ es una genodermatosis que se transmite con un patrón hereditario autosómico dominante con penetrancia variable. En el año 2013 se detectó que es ocasionada por mutaciones en el gen ADAM10, localizado en el cromosoma 15q21.3. ${ }^{2}$

El grupo de desórdenes pigmentarios reticulares genéticos reúne distintas dermatosis caracterizadas por la presencia de manchas hiperpigmentad"as similares a efélides, que tienden a confluir formando un patrón

\footnotetext{
* Dermatóloga, Maestra en Ciencias.

* Especialista en Genética Médica e Investigadora en Ciencias Médicas del Hospital Juárez de México.

$\S$ Dermatopatóloga.

" Dermatóloga.
}

Centro Dermatológico «Dr. Ladislao de la Pascua», SSCDMX. reticular. En su mayoría se ha detectado su asociación con uno o más locus específicos:

- Enfermedad de Dowling-Degos (DD).

- Tipo 1 (KRT5 en 12q13.13).

- Tipo 2 (POFUT1 en 20q11).

- Tipo 3 (17p33.3).

- Tipo 4 (POGLUTI en 3q13).

- Discromatosis simétrica hereditaria o acropigmentación reticulada de Dohi (ADAR en 1q21).

- Enfermedad de Galli-Galli (KRT5 en 12q13.13).

- Síndrome de Haber.

- Acropigmentación reticulada de Kitamura. ${ }^{2}$

Existen casos publicados de una acropigmentación reticulada indistinguible de la ARK en pacientes con la enfermedad de Dowling-Degos (DD), por lo

Citar como: Olguín-García MG, Palacios-Reyes MC, González-González M, Marmolejo-Chavira M. Acropigmentación reticulada de Kitamura. Rev Cent Dermatol Pascua. 2020; 29 (1): 10-15. https://dx.doi.org/10.35366/96264 
que se ha considerado que se trata de una misma entidad con cuadros clínicos distintos, especialmente en el caso de DD tipo 4. ${ }^{3,4}$ Asimismo, algunos autores sugieren que la clasificación de los desórdenes pigmentarios reticulares genéticos sea nuevamente considerada un gran espectro del mismo síndrome ${ }^{5}$ sin embargo, con la finalidad de rebatir lo anterior, se han buscado intencionadamente mutaciones en el locus KRT5 en pacientes con ARK, sin encontrarse presentes, lo cual sostiene que se trata de dos patologías distintas. ${ }^{6,7}$

\section{EPIDEMIOLOGÍA}

Se calcula una prevalencia menor de uno en 100,000 . Existen pocos casos publicados en la literatura. Hasta el momento sólo hay 130 casos publicados. $^{8}$

\section{ETIOLOGÍA}

\section{ADAM10}

El gen ADAM10 contiene 16 exones que codifica la desintegrina y metaloproteinasa 10 de la familia ADAM, la cual incluye más de 40 de proteasas. ADAM10 se encuentra en todos los sustratos de la epidermis y participa en el recambio de dominios extracelulares, incluyendo L1-CAM, CD44, E-cadherina, N-cadherina, IL-6R y CD30 así como en la vía NOTCH. Se ha estudiado su participación en distintas patologías como psoriasis, eccema, y cáncer de piel. ${ }^{9}$ Se ha demostrado que las mutaciones en ADAM10 en ratones originan manchas hiperpigmentadas similares a lentigos. ${ }^{10}$ Hasta el momento, se ha estudiado principalmente en alteraciones del sistema nervioso, como en la enfermedad de Alzheimer. En esta última se han detectado mediante

Tabla 1: Características de desórdenes pigmentarios reticulares genéticos.

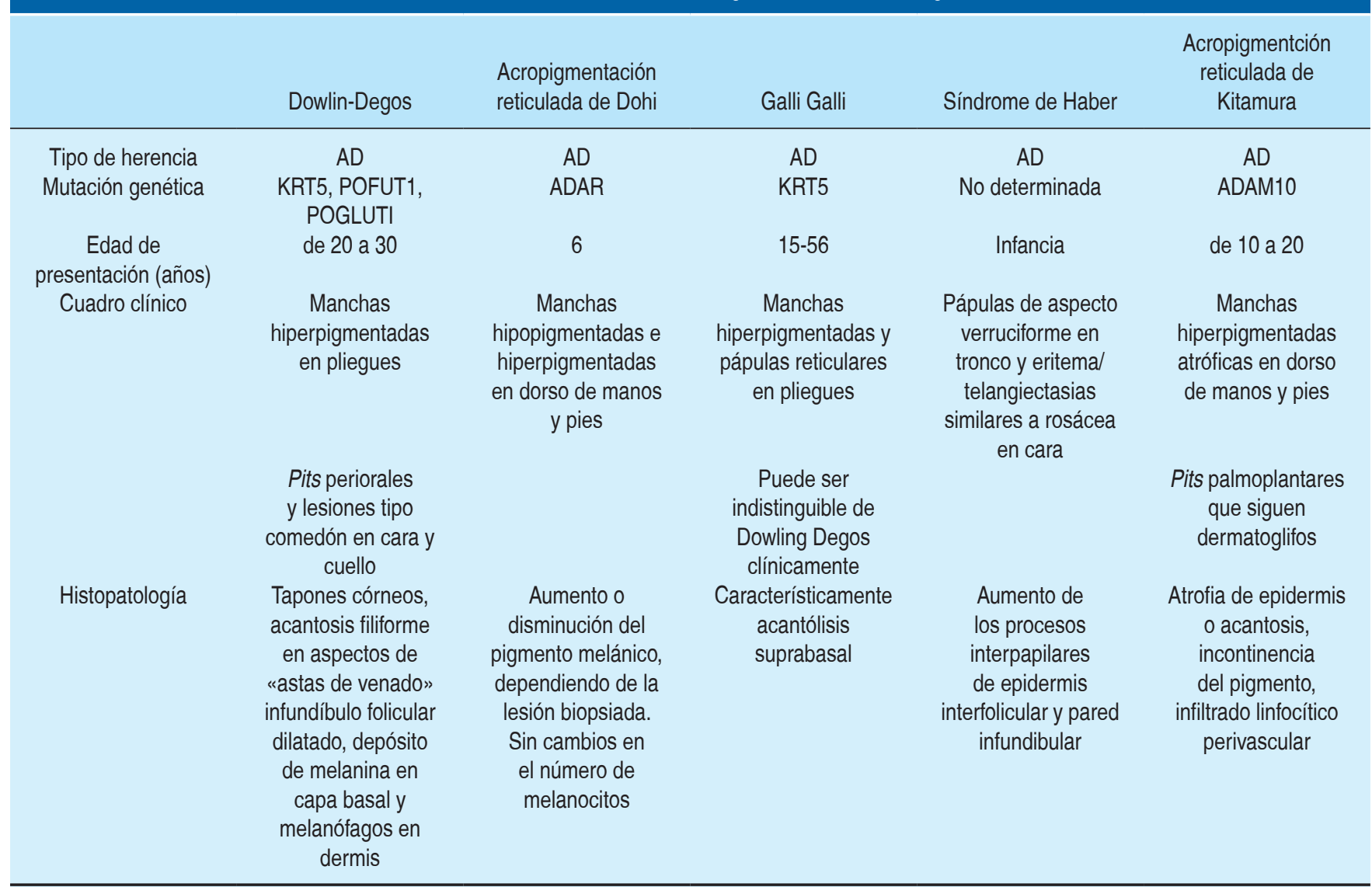

$\mathrm{AD}=$ Autosómico dominante.

Tomado de: Müller CS, Tremezaygues L, Pföhler C, Vogt T. The spectrum of reticulate pigment disorders of the skin revisited. Eur J Dermatol. 2012; 22: 596-604. 
Figura 1:

Aspecto general de la dermatosis en dorso de manos.

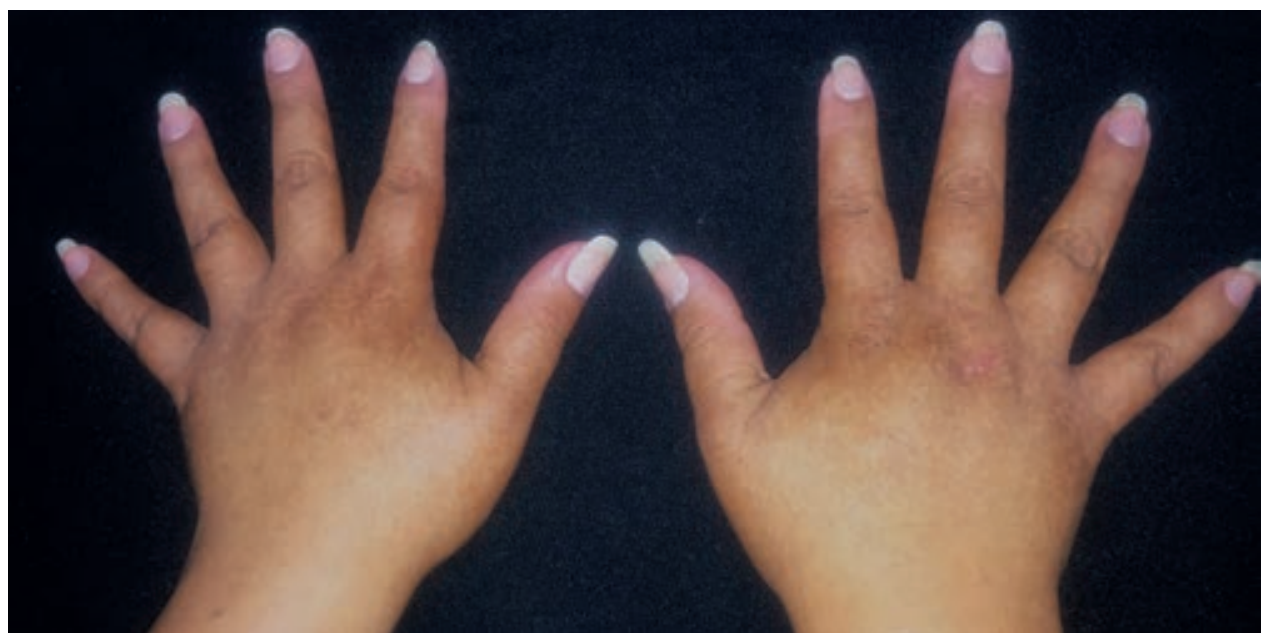

secuenciación cinco mutaciones distintas. ${ }^{2} \mathrm{~A}$ pesar de que se han descrito mutaciones en ADAM10 en pacientes con AKT y bloqueo de las proteasas ADAM, reprimen la melanogénesis, no se conoce la acción de ADAM10 en melanogénesis.

\section{PMEL17 o gp100}

Hasta el momento, mediante estudios in vitro se ha demostrado que la glicoproteína transmembrana PMEL17, también denominada gp100, sufre proteólisis para dar lugar a proteínas fibrilares que forman la matriz celular, sitio de depósito de melanina en los melanosomas, proceso que es regulado por ADAM. Adicionalmente, la inhibición in vitro de la actividad de ADAM17 en melanocitos resulta en menor contenido de melanina y número de melanosomas. Con base en esto, se propone que su función se relaciona más a la distribución11 y al transporte de melanosomas hacia los queratinocitos que a la formación de los melanosomas. ${ }^{9}$

\section{CUADRO CLÍNICO}

Se manifiesta como una dermatosis diseminada a extremidades, de las que afecta dorso de manos y pies, bilateral y simétrica, constituida por pequeñas (0.5-5.0 $\mathrm{mm}$ ) manchas hiperpigmentadas color café en distintos tonos, que asientan sobre áreas de piel atrófica, de evolución crónica y asintomática. Con el paso de los días se va incrementando la intensidad del pigmento y el número hasta comprometer antebrazos, codos y piernas. En forma característica, las palmas y plantas desarrollan pits milimétricos que interrumpen el trayecto de los dermatoglifos. ${ }^{11}$

\section{DIAGNÓSTICO}

Se realiza con base en la correlación clínico histopatológica. Es de suma importancia realizar un interrogatorio minucioso acerca de los antecedentes familiares para determinar si existe un patrón de herencia. El diagnóstico diferencial debe hacerse tomando en cuenta el resto de los desórdenes pigmentarios reticulares genéticos (Tabla 1).

\section{Auxiliares diagnósticos:}

1. Dermatoscopía: Se observa una red fina de pigmento en ausencia de manchas hipopigmentadas y depresiones puntiformes en palmas y plantas. ${ }^{12}$

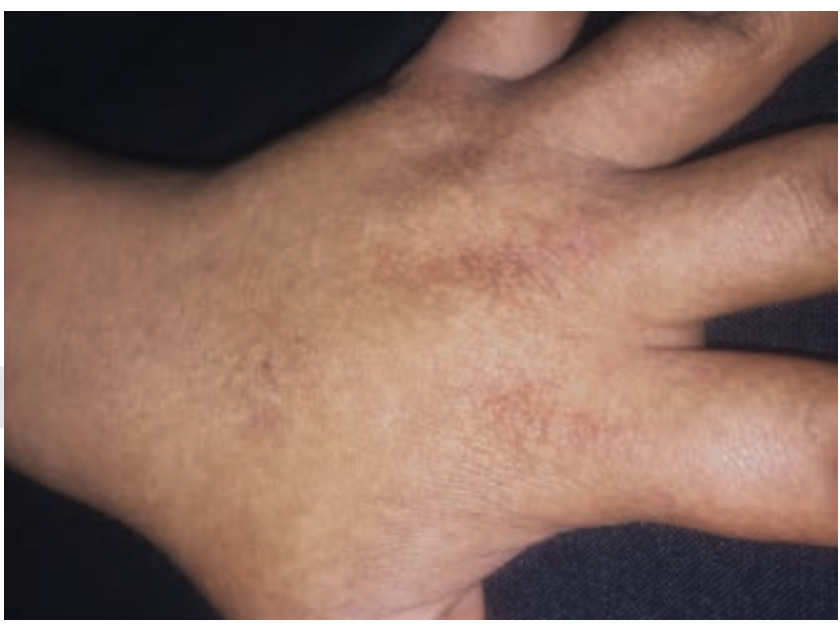

Figura 2: A mayor detalle, manchas hiperpigmentadas reticuladas en dorso de manos. 


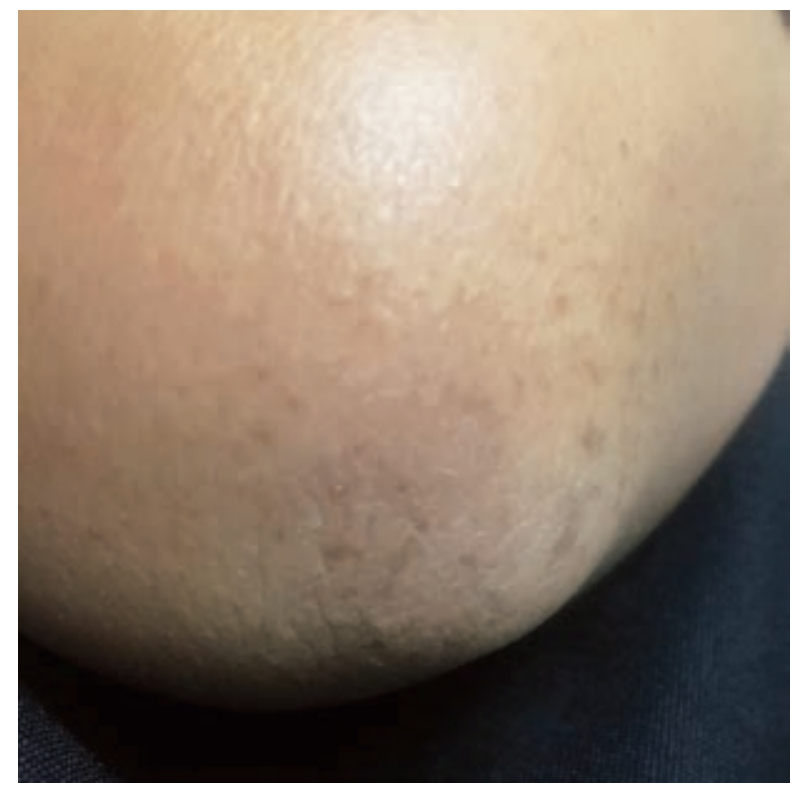

Figura 3: Manchas hiperpigmentadas deprimidas en codos.

2. Huella palmar: Útil para evidenciar las depresiones puntiformes palmoplantares que característicamente interrumpen a los dermatoglifos. ${ }^{12}$

\section{HISTOPATOLOGÍA}

En la epidermis se observa hiperqueratosis ortoqueratósica, atrofia, elongación de los procesos interpapilares, caída del pigmento e infiltrado linfocitario perivascular. Esta última característica, aunque también se observa en la enfermedad de Dowling-Degos, es menos evidente. En la microscopía electrónica se aprecia un número normal de melanocitos, aunque con incremento de los melanosomas en las biopsias tomadas de las manchas. ${ }^{13}$

\section{TRATAMIENTO}

No existe uno como tal. Kameyama utilizó ácido azeláico dos veces al día en cara y una de las rodillas de una paciente, observando mejoría de las manchas del lado tratado así como en las manchas de la cara al término de dos meses, sin efectos adversos reportados. ${ }^{14}$

Fahad y colaboradores comunicaron disminución de $50 \%$ de las lesiones de ARK luego de dos sesiones con láser $\mathrm{Q}$-switched alexandrite, sin cambios a los dos años del tratamiento. ${ }^{15}$ Igualmente J. Lee informó buenos resultados con el láser Q-switched Nd: YAG durante siete sesiones, sin recidiva a los 10 años del tratamiento y sin efectos adversos. ${ }^{16}$

\section{CASO CLÍNICO}

Se trata de paciente del sexo femenino, de 29 años de edad, originaria y residente de la Ciudad de México, dedicada a la docencia, casada, quien acude al Centro Dermatológico «Dr. Ladislao de la Pascua» refiriendo haber notado desde los 16 años la presencia de manchas color café en el dorso de las manos y pies (Figura 1), las cuáles fueron incrementando su tonalidad y su número de manera ascendente, involucrando antebrazos y codos.

Entre sus antecedentes heredofamiliares refiere únicamente la presencia de «manchas» no especificadas en manos de su abuela materna. Dos hijos de cinco y un año respectivamente, aparentemente sanos. Resto de los antecedentes negativos.

A la exploración física se observa dermatosis diseminada a las extremidades, de las que compromete codos y ambas caras de antebrazos así como caras dorsales de carpos y falanges (Figuras 2 y 3). De las extremidades inferiores afecta dorso de pies, bilateral y simétrica. Es una dermatosis constituida por numerosas manchas de color café claro a oscuro, bien delimitadas, confluentes en un patrón reticulado, ligeramente deprimidas a la palpación (Figura 4). De evolución crónica y asintomática. Al observar intencionadamente las palmas se aprecian depresiones puntiformes que siguen e interrumpen los dermatoglifos. Para evidenciar con mayor exactitud los hoyuelos se aplicó tinta china con posterior lavado de las palmas (Figura 5).

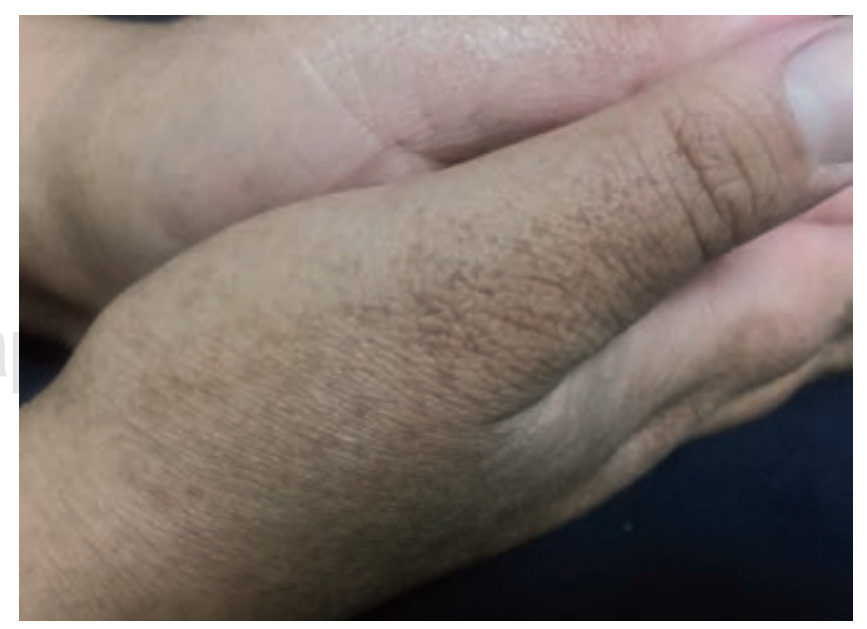

Figura 4: Aspecto reticular de las lesiones, algunas asentadas sobre atrofia. 


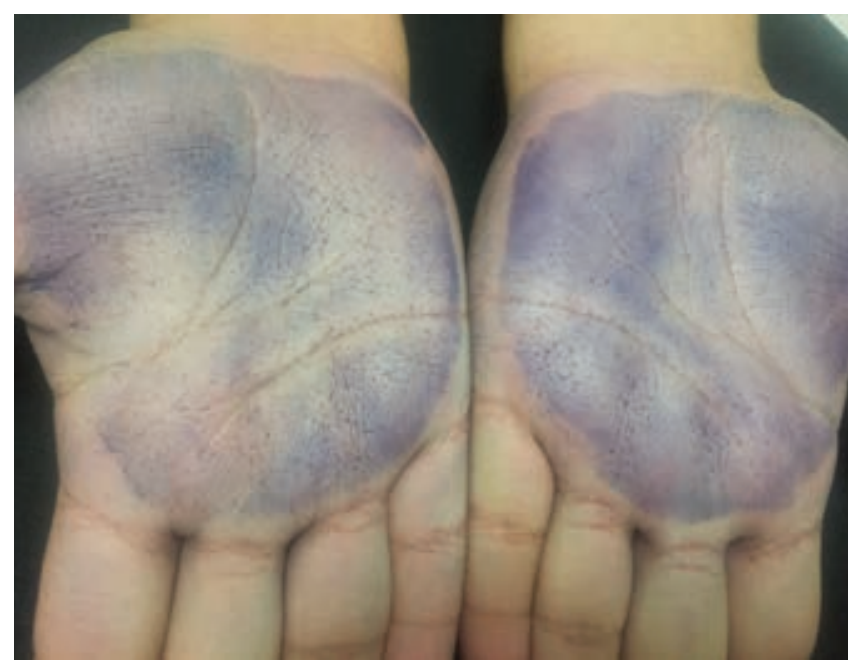

Figura 5: Prueba de «tinta lavada» para evidenciar «pits» palmares.

Las manifestaciones descritas son consistentes con acropigmentación reticulada de Kitamura (ARK), por lo cual se realiza una biopsia incisional de lesiones pigmentadas para confirmar el diagnóstico.

El estudio histopatológico muestra hiperqueratosis ortoqueratósica, elongación de los procesos interpapilares, hiperpigmentación de la capa basal e infiltrados linfocitarios perivasculares en la dermis superficial, datos compatibles con la ARK (Figuras 6 y 7).

Se indicó iniciar tratamiento con ácido glicólico al 10\% en dorso de manos y pies, y filtro solar cada cuatro horas en zonas expuestas. Debido a que es un padecimiento autosómico dominante que inicia entre los 10 y 20 años de edad, se comentó la posibilidad de heredarlo a sus dos hijos con un riesgo de $50 \%$. Se realizó una explicación amplia del padecimiento, la cual asimiló y le brindó tranquilidad. Desde que se realizó el diagnósico, acude regularmente a control, con evolución estable y sin grandes cambios.

\section{COMENTARIO}

El motivo de comunicación del caso descrito en este artículo es lo poco frecuente de su presentación dentro de la consulta dermatológica en general, ${ }^{8} \mathrm{con}$ menos de 200 reportes en la literatura internacional, siendo éste el primer caso mexicano descrito. Probablemente se deba a que este grupo de enfermedades es subdiagnósticado, ya que la ARK es una entidad de comportamiento benigno, asintomática, por lo cual los pacientes difieren acudir a consulta. En este caso en especial, para la paciente fue alta- mente satisfactorio conocer la causa y el pronóstico del padecimiento.

Aún existe controversia en la etiopatogenia y relación entre las entidades que conforman el grupo de desórdenes pigmentarios reticulares genéticos. Aún existe controversia en la etiopatogenia y relación entre las entidades que conforman el grupo de desórdenes pigmentarios reticulares genéticos. Sin embargo, se han determinado sus bases genéticas por separado como entidades monogénicas, aunque representan variantes de una misma entidad clínico-histopatológica. ${ }^{17}$ Aunque

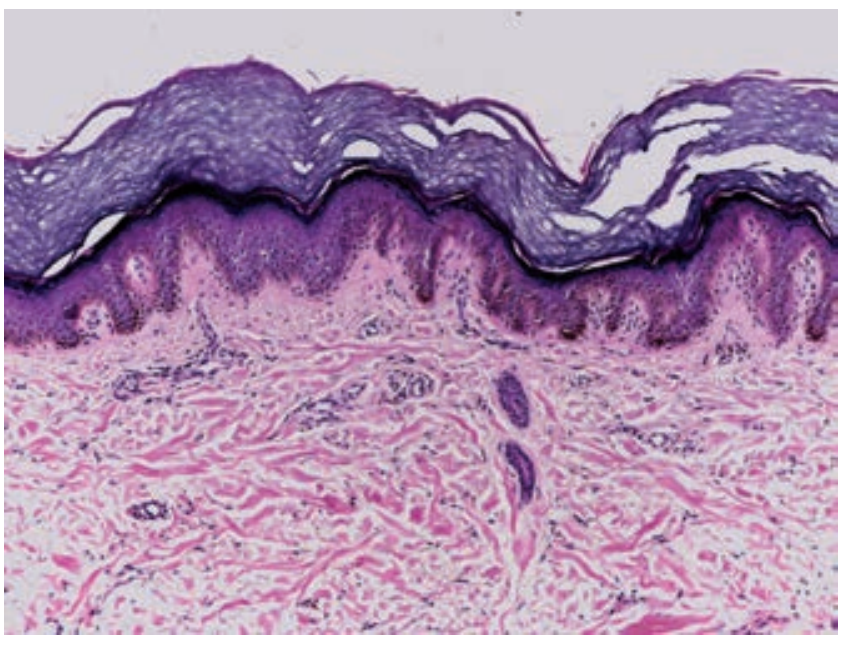

Figura 6: Epidermis con hiperqueratosis ortoqueratósica, elongación de los procesos interpapilares y zonas de hiperpigmentación de la capa basal (H\&E 10x).

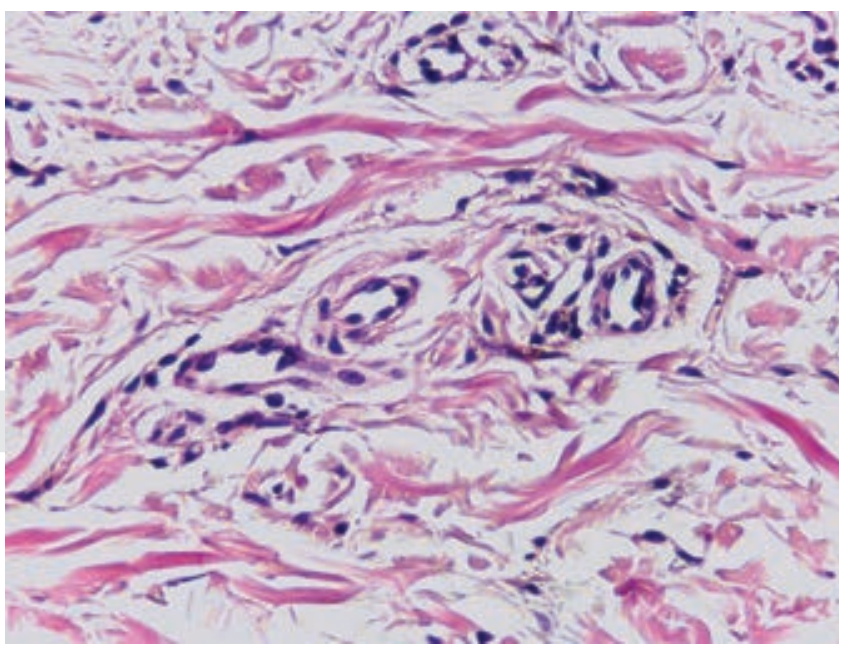

Figura 7: En dermis superficial hay caída de pigmento melánico y discretos infiltrados linfocitarios perivasculares (H\&E 40x). 
existen múltiples reportes de sobreposición entre este espectro, este caso únicamente muestra datos clínicos e histopatológicos de ARK. Característicamente, la ARK es la única que presenta pits palmares y atrofia de las lesiones pigmentadas, ambos presentes en el caso motivo de esta publicación. La detección de los pits palmares es importante para confirmar el diagnóstico. Su presencia puede ser poco perceptible, por lo que recomendamos realizar su búsqueda intencionada con lo que hemos denominado «huella lavada», que consiste en aplicar tinta en las palmas o plantas y enjuagar con agua, sin tallar, de tal manera que la tinta permanezca en las líneas palmares y en los hoyuelos, haciéndolos más evidentes. Esto puede ser de utilidad también en otras patologías ajenas al grupo de desórdenes pigmentarios reticulares como el síndrome de Gorlin-Goltz. ${ }^{18}$

Aún queda mucho por descubrir del comportamiento genético y sobreposición clínica en este grupo de padecimientos así como sus posibilidades terapéuticas, aunque actualmente se puede ofrecer al paciente un diagnóstico y explicación amplia sobre su padecimiento, lo cual resulta de suma importancia para lograr una adecuada calidad de vida en cada uno de ellos.

\section{BIBLIOGRAFÍA}

1. Kitamura K ASNS HIFU shikisoijo. RNHH. No Title. 1943; 8: 4.

2. OMIM. Reticulate acropigmentation of Kitamura (RAK). p. \#615537.

3. Al Hawsawi K, Al Aboud K, Alfadley A, Al Aboud D. Reticulate, acropigmentation of Kitamura \& Dowling Degos disease overlap: a case report. Int J Dermatol. 2002; 41: 518-520.

4. Rathoriya S, Soni SL, Asati D. Dowling-Degos disease with reticulate acropigmentation of Kitamura: extended spectrum of a single entity. Indian Dermatol Online J. 2016; 7: 32-35.

5. Müller CSL, Pföhler C, Tilgen W. Changing a concept-controversy on the confusing spectrum of the reticulate pigmented disorders of the skin. J Cutan Pathol. 2009; 36: 44-48.

6. Kono M, Sugiura K, Suganuma M, Hayashi M, Takama H, Suzuki T et al. Whole-exome sequencing identifies ADAM10 mutations as a cause of reticulate acropigmentation of Kitamura, a clinical entity distinct from Dowling-Degos disease. Hum Mol Genet. 2013; 22: 3524-3533.

7. Kono M, Suganuma M, Takama H, Suzuki T, Matsunaga K, Tomita Y et al. Dowling-Degos disease is genetically and clinico-pathologically distinct from reticulate acropigmentation of Kitamura, further confirmation. J Dermatol Sci. 2016; 84: e81.

8. Prevalence and incidence of rare diseases: bibliographic data. 2017: 1-74. Orphadata databese, www.orphadata.org.

9. Kawaguchi M, Hearing VJ. The roles of ADAMs family proteinases in skin diseases. Enzyme Res. 2011; 2011: 1-9.

10. Kawaguchi M, Hozumi Y, Suzuki T. ADAM protease inhibitors reduce melanogenesis by regulating PMEL17 processing in human melanocytes. J Dermatol Sci. 2015; 78: 133-142.

11. Okamura K, AbeY, ArakiY, HozumiY, Kawaguchi M, Suzuki T. Behavior of melanocytes and keratinocytes in reticulate acropigmentation of Kitamura. Pigment Cell Melanoma Res. 2016; 29: 243-246.

12. Koguchi H, Ujiie H, Aoyagi S, Osawa R, Shimizu H. Characteristic findings of handprint and dermoscopy in reticulate acropigmentation of Kitamura. Clin Exp Dermatol. 2014; 39: 85-87.

13. Calonje JE, Brenn T, Phillip MA. McKee's pathology of the skin. 4th ed. China: Saunders; 2011. p. 1906.

14. Kameyama K, Morita M, Sugaya K, Nishiyama S, Hearing VJ. Treatment of reticulate acropigmentation of Kitamura with azelaic acid. An immunohistochemical and electron microscopic study. J Am Acad Dermatol. 1992; 26: 817-820.

15. Fahad AS, Shahwan HA, Dayel SB. Treatment of reticulated acropigmentation of Kitamura with Q-switched alexandrite laser. Int J Dermatol. 2011; 50: 1150-1152.

16. Hyung Lee J, Hee Lee J, Heung Lee J. A case of reticulate acropigmentation of Kitamura treated with 532-nm Q-Switched Nd: YAG Laser: 10 years of follow-up observation. Ann Dermatol. 2014; 26: 783-785.

17. Wu YH, Lin YC. Generalized Dowling-Degos disease. J Am Acad Dermatol. 2007; 57: 327-334.

18. Mehta D, Raval N, Patadiya H, Tarsariya V. Gorlin-Goltz syndrome. Ann Med Health Sci Res. 2014; 4: 279-282.

Correspondencia:

Dra. María Guadalupe Olguín García

Dr. Vértiz Núm. 464 Esq. Eje 3 Sur,

Col. Buenos Aires, 06780,

Alcaldía Cuauhtémoc, CDMX.

Tel. 5555196351.

E-mail: olguingog@yahoo.com.mx 